\title{
Cuatro nuevos registros de hongos micorrizógenos arbusculares (Glomeromycota) asociados con Agave karwinskii y A. angustifolia (Agavaceae) de Oaxaca, México
}

\section{Four new records of arbuscular mycorrhizal fungi (Glomeromycota) associated with Agave karwinskii and A. angustifolia (Agavaceae) in Oaxaca, Mexico}

\author{
Eduardo Chimal-Sánchezı,3 (D), Irma Reyes Jaramillol (D), Jose Yabin Salmerón-Castrol (D), Nancy Vázquez-Pérez² (D), \\ Lucía Varela-Fregoso'
}

1 Universidad Autónoma Metropolitana, Departamento de Biología, División de Ciencias Biológicas y de la Salud, Unidad Iztapalapa, 09340 Cd. Mx., México.

2 Universidad Autónoma del Estado de Morelos, Centro de Investigación en Biodiversidad y Conservación, Cuernavaca, Morelos, México.

3 Autor para la correspondencia: chimalse31@xanum.uam.mx, chimalse31@gmail.com

Recibido: 12 de marzo de 2018 Revisado: 17 de abril de 2018.

Aceptado: 23 de mayo de 2018 Primero en línea: 21 de agosto de 2018.

Publicado: 3 de octubre de 2018.

Citar como: Chimal-Sánchez, E., I. Reyes Jaramillo, J. Y. Salmerón-Castro, N. Vázquez-Pérez y L. Varela-Fregoso. 2018. Cuatro nuevos registros de hongos micorrizógenos arbusculares (Glomeromycota) asociados con Agave karwinskii y A. angustifolia (Agavaceae) de Oaxaca, México. Acta Botanica Mexicana 125: 173-187. DOI: 10.21829/abml25.2018.1356

DOI:

10.21829/abml25.2018.1356

\section{Resumen:}

Antecedentes y Objetivos: Los agaves son un recurso natural apreciado por la gente de Oaxaca como fuente de alimento y para la producción de mezcal; sin embargo, hay escasa información de los hongos micorrizógenos arbusculares (HMA) asociados a ellos. El objetivo de este estudio es contribuir al conocimiento taxonómico de los HMA en México con cuatro nuevos registros; en particular, en agaves mezcaleros de la región semiárida de Oaxaca.

Métodos: En nueve sitios de los Valles Centrales y de la Sierra Sur de Oaxaca se recolectó suelo rizosférico de tres plantas de Agave karwinskii y de A. angustifolia para aislar las esporas de HMA y realizar preparaciones permanentes con alcohol polivinílico en lactoglicerol (PVLG) y reactivo de Melzer. Con base en caracteres morfológicos de las esporas se realizó la identificación taxonómica. Asimismo, se determinó el ambiente edáfico como la textura, el $\mathrm{pH}$ activo (1:2.5 suelo-agua), el porcentaje de materia orgánica, el fósforo disponible y las bases extraíbles $\mathrm{Ca}^{++}, \mathrm{Mg}^{++}, \mathrm{K}^{+}$y $\mathrm{Na}^{+}$.

Resultados clave: Cuatro especies de Glomeromycota se citan por primera vez para México: Acaulospora minuta, A. papillosa, A. reducta y Paraglomus bolivianum, todas asociadas con la rizósfera de Agave karwinskii y las últimas dos especies también con la de A. angustifolia. Los HMA se registraron en suelos de textura gruesa, ricos en calcio y pobres en fósforo disponible. Las descripciones taxonómicas e ilustraciones de las cuatro especies son presentadas.

Conclusiones: Con estos cuatro nuevos registros, Acaulospora minuta, A. papillosa, A. reducta y $P$. bolivianum, se contabilizan 148 especies de HMA en México. Oaxaca es un estado de alta diversidad florística y centro de diversificación de plantas, entre ellos los agaves; sin embargo, los HMA han sido escasamente estudiados y probablemente su diversidad taxonómica sea importante.

Palabras clave: Acaulospora, Agave, HMA, mezcal, Paraglomus, simbiosis.

\section{ABSTRACT:}

Background and Aims: The agaves are a natural resource appreciated by about people from Oaxaca as food source and for the mescal production; however, there is scarce information the arbuscular mycorrhizal fungi (AMF) associated to them. The objective of this study is to contribute to the taxonomic knowledge of the AMF in Mexico with four new records; in particular, in mescal agaves of the semi-arid region of Oaxaca.

Methods: In nine sites of the Central Valleys and the Southern Highlands of Oaxaca, rhizosphere soil was collected from three plants of Agave karwinskii and A. angustifolia to isolate the spores of AMF, and to make permanent preparations with polyvinyl lacto glicerol (PVLG) and reagent of Melzer. Morphological characters of the spores were used for taxonomic identification. The soil texture, the active $\mathrm{pH}$ (1:2.5 soil-water), the percentage of organic matter, the available phosphorus and the extractable bases $\mathrm{Ca}^{++}, \mathrm{Mg}^{++}, \mathrm{K}^{+}$and $\mathrm{Na}^{+}$were determined.

Key results: Four species of Glomeromycota are cited for the first time from Mexico: Acaulospora minuta, A. papillosa, A. reducta and, Paraglomus bolivianum, all associated with the rhizosphere of Agave karwinskii and, the last two species, also with that of $A$. angustifolia. The AMF were registered in soils of thick texture, rich in calcium and poor in available phosphorus. The taxonomic descriptions and illustrations of the four species are presented.

Conclusions: With these four new records, Acaulospora minuta, A. papillosa, A. reducta and P. bolivianum, 148 species of AMF are known from Mexico. Oaxaca is a state of high floristic diversity and a center of diversification of plants, among them agaves; however, the AMF have been barely studied and their taxonomic diversity is probably important.

Key words: Acaulospora, Agave, AMF, mescal, Paraglomus, symbiosis. 


\section{INTRODUCCIÓN}

Los agaves cultivados o silvestres son parte del paisaje del estado de Oaxaca, en donde Agave angustifolia Haw. y A. karwinskii Zucc. (Agavaceae) son plantas de importancia cultural y económica para sus pobladores, además de formar parte de diversos tipos de vegetación como el matorral xerófilo y el bosque tropical caducifolio. Sus usos son variados, en especial los jugos de tallos y base de hojas cocidos que se utilizan para preparar el mezcal; su elaboración tradicional se ha conservado por generaciones de grupos indígenas de Oaxaca, los cuales identifican a los agaves y al mezcal como un símbolo de identidad cultural (García-Mendoza et al., 2004; Vázquez-Pérez, 2015).

Agave angustifolia crece de manera silvestre en toda la sierra occidental del sur y sureste de México. En Oaxaca es conocido como espadín; por su alta producción en azúcares, tiempo de maduración (5-7 años) y fácil propagación por hijuelos, se ha convertido en el principal maguey cultivado en el estado destinado a la producción de mezcal. También se usan otros agaves silvestres como A. karwinskii que posee distintas variedades y formas morfológicas silvestres y manejadas, se le conoce con diferentes nombres comunes: cuishe, san martín y tobasiche, en los Valles Centrales; mientras que bicuishe y madrecuishe son los nombres empleados en la Sierra Sur de Oaxaca. Esta especie se caracteriza por tener un tallo largo (caulinar) que se utiliza también para la producción del mezcal en forma artesanal a nivel local (García-Mendoza et al., 2004; Vázquez-Pérez, 2015).

Diversas investigaciones indican que los hongos micorrizógenos arbusculares (HMA, Glomeromycota) se asocian con las raíces de agaves, como Agave marmorata Roezl, A. salmiana Otto ex Salm-Dyck (Camargo-Ricalde et al., 2003), A. lechuguilla Torr., A. striata Zucc. (GarcíaSánchez et al., 2008), A. potatorum Zucc. (Carballar-Hernández et al., 2013) y A. angustifolia (Ochoa-Meza et al., 2009). La simbiosis de los HMA con los agaves puede ser de gran importancia para su supervivencia y nutrición en los ecosistemas áridos y semiáridos que habitan, debido a que en estos ambientes se presentan altas temperaturas, poca disponibilidad de agua, fósforo y nitrógeno en el suelo (Montaño et al., 2008). Debido a la relevancia de los agaves mezcaleros y a su simbiosis con los HMA, se han realizado estudios enfocados a determinar la diversidad de estos hongos presentes en los suelos en donde crecen A. karwinskii, A. marmorata y A. potatorum de los Valles Centrales (Carballar-Hernández et al., 2013), así como de A. potatorum en la región Mixteca de Oaxaca (Hernández-Morales et al., 2014), con el fin de utilizar consorcios de HMA potencialmente eficientes para el cultivo de los agaves mezcaleros (Robles-Martínez et al., 2013).

En los Valles Centrales de Oaxaca, Carballar-Hernández (2009) y Carballar-Hernández et al. (2013) registraron en A. marmorata, A. karwinskii y A. potatorum una amplia diversidad de especies de HMA en condiciones naturales; principalmente de las familias Acaulosporaceae y Glomeraceae. Asimismo, reportaron morfoespecies del género Acaulospora Gerd. \& Trappe con caracteres morfológicos diferentes a los de las especies de HMA ya descritas, lo cual sugiere que en el suelo de los agaves hay una diversidad taxonómica de HMA aún desconocida. Así, al estudiar los HMA asociados con $A$. angustifolia y A. karwinskii en los Valles Centrales y en la Sierra Sur de Oaxaca, en la presente investigación, se identificaron tres especies del género Acaulospora y una de Paraglomus J.B. Morton \& D. Redecker no registradas con anterioridad en ecosistemas de México (Alarcón et al., 2012; Montaño et al., 2012), particularmente en agaves (Carballar-Hernández et al., 2013; Robles-Martínez et al., 2013). De esta manera, los objetivos de este estudio son reportar y presentar las descripciones taxonómicas de cuatro nuevos registros de especies de HMA para México, provenientes del estado de Oaxaca, asociados al suelo de dos especies de agaves mezcaleros: A. angustifolia y $A$. karwinskii.

\section{Materiales y Métodos}

El muestreo se realizó en la Región de los Valles Centrales y en la Región de la Sierra Sur de Oaxaca; ambas pertenecen a la Provincia Biogeográfica Sierra Madre del Sur. El clima es semi-seco cálido a semi-cálido (BSh) con lluvias en verano. Los suelos que predominan 
son Regosoles, Leptosoles, Calcisoles y Phaeozems. La vegetación comprende selvas bajas caducifolias y matorrales xerófilos que se encuentran fuertemente alterados $\mathrm{y}$ fragmentados por las actividades agropecuarias (INEGI, 2010).

El muestreo de suelo rizosférico se hizo durante la temporada de secas (enero 2016) en nueve sitios de muestreo (S), cinco en los Valles Centrales (S1 al S5) y cuatro en la Sierra Sur (S6 al S9) (Cuadro 1). En cada uno de ellos se seleccionaron tres plantas diferentes de A. karwinskii o de $A$. angustifolia y, de cada agave, se recolectó una muestra compuesta de suelo a partir de tres sub-muestras de $600 \mathrm{~g} \mathrm{c} / \mathrm{u}$, tomadas alrededor de la base de la planta a una profundidad de 0 a $20 \mathrm{~cm}$. Se colocaron en bolsas de plástico negras para su traslado al Laboratorio de Edafología de la Universidad Autónoma Metropolitana, Iztapalapa (UAM-IZ). Asimismo, se establecieron macetas para la propagación de los HMA con el suelo recolectado (500 g/maceta) en condiciones de invernadero para obtener esporas recién formadas (Stutz y Morton, 1996). Las plantas hospederas fueron maíz (Zea mays L.) y una leguminosa (Leucaena sp.) conocida como guaje. El período de propagación fue de seis meses con riegos cada tercer día.

La extracción de esporas de HMA se hizo por el método de tamizado en húmedo y decantación (Gerdemann y Nicolson, 1963), usando $100 \mathrm{~g}$ de suelo por cada muestra. Para separar las esporas del material mineral y orgánico del suelo se centrifugó en una solución de sacarosa al 60\%. Por medio de un microscopio estereoscópico (Leica Zoom 2000, Nueva York, EUA) se separaron los morfotipos de los HMA, se contabilizaron y se realizaron preparaciones permanentes de las esporas con alcohol polivinílico en lactoglicerol (PVLG) y PVLG con reactivo de Melzer $(1: 1, \mathrm{v} / \mathrm{v})$ como lo recomienda la International Culture Collection of (Vesicular) Arbuscular Mycorrhizal Fungi (INVAM, 2017). De esta manera se observaron al microscopio óptico (Olympus BX41, Tokio, Japón) con contraste de interferencia de Nomarski y se tomaron microfotografías con una cámara Olympus (C5060, Tokio, Japón).
La determinación taxonómica de las especies de HMA se basó en el reconocimiento, comparación y contraste de los caracteres morfológicos de sus esporas y la reacción al reactivo de Melzer de las capas que constituyen la pared. Las esporas se midieron con una reglilla micrométrica acoplada a uno de los oculares del microscopio y su coloración se obtuvo con una fórmula de porcentajes de azul, magenta, amarillo y negro (INVAM, 2017). Esta información se contrastó con las descripciones de las especies de Glomeromycota conocidas y disponibles en "arbuscular mycorrhizal fungi phylogeny (Schüßler, 2017), en el INVAM (2017) y en descripciones e ilustraciones de HMA propuesto por Blaszkowski (2017). Para describir las paredes de las esporas de Acaulospora se siguió la terminología empleada por Oehl et al. (2012); para las esporas de Paraglomus se empleó la terminología de Oehl y Sieverding (2004) y Aragão de Mello et al. (2013). Los ejemplares biológicos de referencia se depositaron en el Herbario de la Escuela Nacional de Ciencias Biológicas (ENCB) (acrónimo de acuerdo con Holmgren et al., 1990) del Instituto Politécnico Nacional (IPN) y respaldos en el laboratorio de Edafología de la Universidad Autónoma Metropolitana, Iztapalapa (UAM-IZ) que están disponibles para su consulta.

Para caracterizar el ambiente edáfico donde crecen los agaves y los HMA se practicaron los siguientes análisis de acuerdo con los procedimientos propuestos en Jackson (1976) y Van Reeuwijk (1999): pH por medio de un potenciómetro (Thermo Scientific, Orion Star, Singapur), en una relación 1:2.5 suelo-agua, la textura por el método de Bouyoucos, la materia orgánica (MOS) por combustión húmeda por el método de Walkley \& Black y el fósforo disponible por el método de Olsen (Olsen et al., 1954). Las bases extraíbles calcio $\left(\mathrm{Ca}^{++}\right)$, magnesio $\left(\mathrm{Mg}^{++}\right)$, sodio $\left(\mathrm{Na}^{+}\right)$y potasio $\left(\mathrm{K}^{+}\right)$se extrajeron con una solución de acetato de amonio $1 \mathrm{~N}, \mathrm{pH} 7$; así el $\mathrm{Ca}^{++} \mathrm{y} \mathrm{Mg}^{++}$fueron valorados por el método de Versenato (EDTA), mientras que el $\mathrm{Na}^{+}$y el K${ }^{+}$se determinaron por medio de flamometría de emisión (Flame Photometer M410, Corning; Halstead, Essex Inglaterra). 
Cuadro 1: Sitios de recolecta de suelo asociado a Agave angustifolia Haw. y Agave karwinskii Zucc. en los Valles Centrales (S1-S5) y Sierra Sur (S6-S9) de Oaxaca, México. * Según la localidad, los pobladores nombran y conocen a Agave karwinskii como cuishe, san martín, tobasiche, bicuishe o madrecuishe; mientras que al Agave angustifolia, como espadín. Metros sobre el nivel del mar (m s.n.m.), pH es el activo relación 1:2.5 (suelo:agua), porcentaje materia orgánica (MO), fósforo disponible (P)(Olsen et al., 1954).

\begin{tabular}{|c|c|c|c|c|}
\hline Sitio & Coordenadas & $\begin{array}{l}\text { Altitud } \\
\text { (m s.n.m.) }\end{array}$ & Especie vegetal & $\begin{array}{l}\text { Características del sitio y condiciones edáficas en donde } \\
\text { crecen los agaves mezcaleros }\end{array}$ \\
\hline S1, San Pedro Totolapan & $\begin{array}{l}16^{\circ} 40^{\prime} 58^{\prime \prime} \mathrm{N} \\
96^{\circ} 18^{\prime} 23^{\prime \prime} \mathrm{O}\end{array}$ & 940 & $\begin{array}{l}\text { Agave karwinskii } \\
\text { (cuishe*) }\end{array}$ & $\begin{array}{l}\text { Matorral xerófilo semi-conservado y poblaciones silvestres de } \\
\text { agave. Suelo franco-arenoso, } \mathrm{pH}: 7.52\left(\mathrm{H}_{2} \mathrm{O}\right), \mathrm{MO}: 2.68 \% \text {, P: } \\
4 \mathrm{mg} \mathrm{kg}^{-1} \text {. }\end{array}$ \\
\hline $\begin{array}{l}\text { S2, km } 50 \text { Carretera Oaxaca } \\
\text { - Ejutla }\end{array}$ & $\begin{array}{l}16^{\circ} 38^{\prime} 31^{\prime \prime} \mathrm{N} \\
96^{\circ} 44^{\prime} 04.2 " \mathrm{O}\end{array}$ & 1508 & $\begin{array}{l}\text { Agave angustifolia } \\
\text { (espadín) }\end{array}$ & $\begin{array}{l}\text { Zona de cultivo abandonada con individuos de } \text { A. angustifolia. } \\
\text { Suelo franco-arenoso, } \mathrm{pH}: 7.19\left(\mathrm{H}_{2} \mathrm{O}\right), \mathrm{MO}: 2.16 \% \text {, } \mathrm{P}: 0.8 \\
\mathrm{mg} \mathrm{kg}^{-1} \text {. }\end{array}$ \\
\hline S3, San Agustín Amatengo & $\begin{array}{l}16^{\circ} 31^{\prime} 05.8^{\prime \prime} \mathrm{N} \\
96^{\circ} 47^{\prime} 08.2^{\prime \prime} \mathrm{O}\end{array}$ & 1387 & $\begin{array}{l}\text { Agave karwinskii } \\
\text { (san martín*) }\end{array}$ & $\begin{array}{l}\text { Vegetación secundaria con poblaciones manejadas de agave. } \\
\text { Suelo arenoso-franco, pH: } 7.28\left(\mathrm{H}_{2} \mathrm{O}\right), \mathrm{MO}: 3.26 \% \text {, P: } 0.7 \\
\mathrm{mg} \mathrm{kg}^{-1} \text {. }\end{array}$ \\
\hline S4, San Agustín Amatengo & $\begin{array}{l}16^{\circ} 31^{\prime} 05.8^{\prime \prime} \mathrm{N} \\
96^{\circ} 47^{\prime} 08.2^{\prime \prime} \mathrm{O}\end{array}$ & 1387 & $\begin{array}{l}\text { Agave karwinskii } \\
\text { (tobasiche*) }\end{array}$ & $\begin{array}{l}\text { Vegetación secundaria con poblaciones de agave, cercas vivas } \\
\text { para delimitar terrenos. Suelo franco-arenoso, } \mathrm{pH}: 7.86 \\
\left(\mathrm{H}_{2} \mathrm{O}\right), \mathrm{MO}: 2.51 \% \text {, P: } 0.47 \mathrm{mg} \mathrm{kg}^{-1} \text {. }\end{array}$ \\
\hline $\begin{array}{l}\text { S5, km } 89 \text { Carretera Ejutla - } \\
\text { Miahuatlán }\end{array}$ & $\begin{array}{l}16^{\circ} 22^{\prime} 23.1^{\prime \prime} \mathrm{N} \\
96^{\circ} 39^{\prime} 07.6^{\prime \prime} \mathrm{O}\end{array}$ & 1518 & $\begin{array}{l}\text { Agave karwinskii } \\
\quad \text { (bicuishe*) }\end{array}$ & $\begin{array}{l}\text { Sitio perturbado con pastoreo evidente y poblaciones toleradas } \\
\text { de agave. Suelo franco-arenoso, } \mathrm{pH}: 8.06, \mathrm{MO}: 4.3 \% \text {, } \mathrm{P}: 0.53 \\
\mathrm{mg} \mathrm{kg}^{-1} \text {. }\end{array}$ \\
\hline $\begin{array}{l}\text { S6, San Luis Amatlán, } \\
\text { Miahuatlán }\end{array}$ & $\begin{array}{l}16^{\circ} 22^{\prime} 28.7 " \mathrm{~N} \\
96^{\circ} 39^{\prime} 11.1^{\prime \prime O}\end{array}$ & 1525 & $\begin{array}{l}\text { Agave karwinskii } \\
\text { (madrecuishe*) }\end{array}$ & $\begin{array}{l}\text { Matorral xerófilo semi-conservado con acacias, Leucaena } \\
\text { (guajes), opuntias y poblaciones de agaves en cercos vivos. } \\
\text { Suelo franco-arenoso, pH: } 8.02, \mathrm{MO}: 5.72 \%, \mathrm{P}: 1.2 \mathrm{mg} \mathrm{kg}^{-1} \text {. }\end{array}$ \\
\hline $\begin{array}{l}\text { S7, San Luis Amatlán, } \\
\text { Miahuatlán }\end{array}$ & $\begin{array}{l}16^{\circ} 22^{\prime} 28.7^{\prime \prime} \mathrm{N} \\
96^{\circ} 39^{\prime} 11.1 " \mathrm{O}\end{array}$ & 1525 & $\begin{array}{c}\text { Agave angustifolia } \\
\text { (espadín) }\end{array}$ & $\begin{array}{l}\text { Matorral xerófilo alterado con poblaciones silvestres de } \\
\text { acacias, guajes y agaves cultivados. Suelo franco-arenoso, } \\
\text { pH: } 8.04, \mathrm{MO}: 4.78 \% \text {, P: } 2.0 \mathrm{mg} \mathrm{kg}^{-1} \text {. }\end{array}$ \\
\hline $\begin{array}{l}\text { S8, San Luis Amatlán, } \\
\text { Miahuatlán }\end{array}$ & $\begin{array}{l}16^{\circ} 22^{\prime} 23.4^{\prime \prime} \mathrm{N} \\
96^{\circ} 28^{\prime} 39.9^{\prime \prime O}\end{array}$ & 1555 & $\begin{array}{l}\text { Agave karwinskii } \\
\text { (bicuishe*) }\end{array}$ & $\begin{array}{l}\text { Matorral xerófilo y selva baja caducifolia semi-conservada } \\
\text { con leguminosas espinosas, pastos y agaves silvestres. Suelo } \\
\text { franco-arenoso, pH: } 7.96, \mathrm{MO}: 5.4 \% \text {, P: } 4.2 \mathrm{mg} \mathrm{kg}^{-1} \text {. }\end{array}$ \\
\hline $\begin{array}{l}\text { S9, San Luis Amatlán, } \\
\text { Miahuatlán }\end{array}$ & $\begin{array}{l}16^{\circ} 22^{\prime} 23.4^{\prime \prime} \mathrm{N} \\
96^{\circ} 28^{\prime} 39.9^{\prime \prime} \mathrm{O}\end{array}$ & 1555 & $\begin{array}{l}\text { Agave karwinskii } \\
\text { (madrecuishe*) }\end{array}$ & $\begin{array}{l}\text { Matorral xerófilo y selva baja caducifolia semi-conservada con } \\
\text { leguminosas, gramíneas y agaves silvestres. Suelo franco- } \\
\text { arenoso, } \mathrm{pH}: 8.0, \mathrm{MO}: 4.09 \%, \mathrm{P}: 0.25 \mathrm{mg} \mathrm{kg}^{-1} \text {. }\end{array}$ \\
\hline
\end{tabular}

\section{TAXONOMÍA}

Acaulospora minuta Oehl, Tchabi, Hount., Palenz., I.C. Sánchez \& G.A. Silva, J. Appl. Bot. Food Qual. 84: 214. 2011. TIPO: Benin. Sur de Guinea, $08^{\circ} 20^{\prime} \mathrm{N}$; 01 51 'E, sabana natural, XII.2006, F. Oehl F0366 (holotipo: 3343 (ZT), isotipo: 3344 (ZT)). MycoBank MB 561834. Figs. 1A-D.

Esporas solitarias en el suelo dispuestas lateralmente sobre restos del sáculo esporífero que es hialino, espo- ras color anaranjado claro (0/10/40/0), globosas, 150-170 $\mu \mathrm{m}$ de diámetro, al romperse la pared de la espora se divide en tres grupos, pared externa (OW), intermedia (MW) e interna (IW); la pared más externa (OW) con tres capas (OWL1-L3), ninguna de las capas reaccionó al reactivo de Melzer, juntas tienen un grosor de $4.8 \mu \mathrm{m}$; OWL1 evanescente, ausente en esporas maduras; OWL2 color anaranjado claro $(0 / 10 / 40 / 0)$, laminada, $3.1 \mu \mathrm{m}$ de grosor, cubierta de diminutas depresiones regulares, 1.18-1.52 $\mu \mathrm{m}$ de diámetro, profundidad menor a $1 \mu \mathrm{m}$, separación entre 0.9 y $1.8 \mu \mathrm{m}$, de distribución homogénea, OWL3 
similar en color a OWL2, menor a $1 \mu \mathrm{m}$ de grosor; la pared intermedia (MW) con dos capas hialinas (MWL1L2), no reaccionaron al reactivo de Melzer, semiflexibles, fuertemente adheridas que hace pensar que se trata de una sola capa, ambas capas miden 1.3-1.5 $\mu \mathrm{m}$ de grosor, la pared interna (IW) con tres capas (IWL1-L3) hialinas, semiflexibles, en conjunto miden $2.57 \mu \mathrm{m}$; IWL1 menos de $1 \mu \mathrm{m}$ de grosor con pequeñas granulaciones sobre su superficie; IWL2 $1.3 \mu \mathrm{m}$ de grosor, reaccionó al reactivo de Melzer cambiando su coloración de hialina a rosada (0/30/20/0); IWL3 midió menos de $0.5 \mu \mathrm{m}$ de grosor; cicatriz subcircular, $13.5 \mu \mathrm{m}$ de ancho y $15.5 \mu \mathrm{m}$ de largo; el borde de su circunferencia engrosado y ornamentado con las diminutas depresiones.

Hábitat y distribución: Acaulospora minuta solo se había reportado de Benin, del Oeste Sub-Sahariano de
África en el suelo de siete sitios de sabana natural y de zonas de cultivo con Dioscorea spp. En invernadero $A$. minuta formó simbiosis micorrízica con plantas como $D$. cayenensis Lam., D. rotundata Poir. y Sorghum bicolor Pers. (Oehl et al., 2011). Ahora también se distribuye en el sureste mexicano en el estado de Oaxaca, en la rizósfera de $A$. karwinskii.

Material examinado: MÉXICO. Oaxaca, municipio San Agustín Amatengo (S3), Valles Centrales de Oaxaca, 1387 m s.n.m., $16^{\circ} 31^{\prime} 05.8^{\prime \prime} \mathrm{N}, 96^{\circ} 47^{\prime} 08.2^{\prime \prime} \mathrm{O}$, en la rizósfera de Agave karwinskii (san martín): suelo arenoso-franco ( $79.2 \%$ arena, $16.4 \%$ limo y $4.4 \%$ arcilla), neutro (pH: 7.28, $\mathrm{H}_{2} \mathrm{O}$ ), medianamente rico en $\mathrm{MO}(3.26 \%$ ), muy pobre en fósforo disponible $\left(0.7 \mathrm{mg} \mathrm{kg}^{-1}\right)$, con altos contenidos de calcio $\left(15\right.$ cmoles $\left._{(+)} \mathrm{kg}^{-1}\right)$ y magnesio $(7.5$ cmoles $\left._{(+)} \mathrm{kg}^{-1}\right)$ y bajos en potasio $\left(0.29\right.$ cmoles $\left._{(+)} \mathrm{kg}^{-1}\right)$ y so-
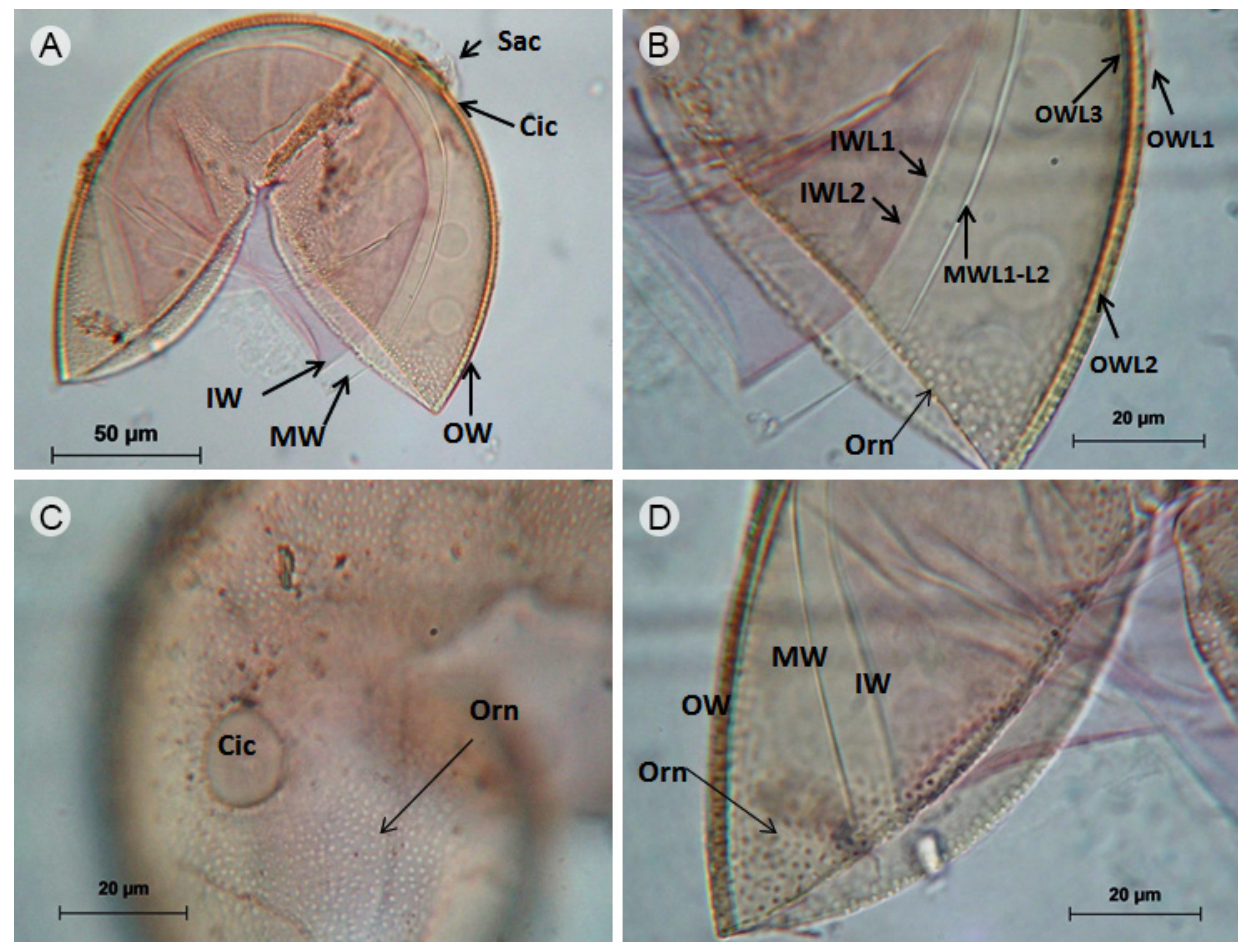

Figura 1: Acaulospora minuta Oehl, Tchabi, Hount., Palenz., I.C. Sánchez \& G.A. Silva. A-D. esporas en reactivo de Melzer, A. espora con una cicatriz (Cic), restos del sáculo (Sac) y pared de la espora que se divide en externa (OW), intermedia (MW) e interna (IW); B. OW constituida de tres capas (OWL1-L3) con ornamentación (Orn) en OWL2, MW con dos capas (MWL1-L2), IW con tres capas (IWL1-L3), únicamente la capa IWL2 reaccionó al reactivo de Melzer, IWL3 al medir menos de $0.5 \mu \mathrm{m}$ de grosor es una capa difícil de observar por estar fuertemente adherida a IWL2; C. detalle de la cicatriz (Cic) y de la ornamentación (Orn) de la espora en la pared laminada; D. detalle de la ornamentación (Orn) que consiste en depresiones sobre la capa laminada de OW, mientras que las capas intermedias (MW) e internas (IW) son hialinas y lisas; solo la capa más interna de IW reaccionó ligeramente al reactivo de Melzer. 
dio $\left(0.14\right.$ cmoles $\left._{(+)} \mathrm{kg}^{-1}\right)$, 20.I.2016, J. Y. Salmerón-Castro $008 a$ (ENCB).

Comentarios taxonómicos: El género Acaulospo$r a$ está constituido por 55 especies formalmente descritas; de ellas, casi $65 \%$ presenta esporas con algún tipo de ornamentación en la pared laminada (Oehl et al., 2012; Schüßler, 2017) como son espinas (A. spinosa Walker \& Trappe), tubérculos (A. tuberculata Janos $\&$ Trappe), depresiones (A. scrobiculata Trappe), verrugas (A. ignota Blaszk., Góralska, Chwat \& Goto), pliegues cerebriformes (A. rehmii Sieverd. \& S. Toro) o una doble ornamentación que consiste de espinas o depresiones encerradas por un retículo (A. bireticulata F.M. Rothwell \& Trappe o A. reducta Oehl, B.T. Goto $\&$ C.M.R. Pereira). Debido a que A. minuta posee esporas con una pared laminada cubierta con depresiones diminutas $(<2 \mu \mathrm{m}$ de diámetro) distribuidas de forma regular, puede ser diferenciada de $A$. cavernata Blaszk., A. excavata Ingleby \& C. Walker, A. foveata Trappe \& Janos, A. reducta y A. verna Blaszk., que presentan depresiones de mayor tamaño ( $>4 \mu \mathrm{m}$ de diámetro) en la capa laminada. Acaulospora minuta difiere de $A$. baetica Palenz., Oehl, Azcón-Aguilar \& G.A. Silva, $A$. herrerae E. Furrazola, B.T. Goto, G.A. Silva, Sieverd. \& Oehl, A. lacunosa J.B. Morton, A. nivalis Oehl, Palenz., Sánchez-Castro, G.A. Silva \& Sieverd., A. scrobiculata y A. sieverdingii Oehl, Sýkorová, Blaszk. \& G.A. Silva, porque las depresiones en las capas laminadas son más grandes e irregulares en comparación con las depresiones regulares diminutas en diámetro de $A$. minuta. Asimismo, difiere de A. alpina Oehl, Sýkorová $\&$ Sieverd., que presenta depresiones regulares sobre la capa laminada, pero que son de forma cónica y más grandes y espaciadas que en $A$. minuta. Otras especies como A. paulinae Blasz. o A. punctata Oehl, Palenz., Sánchez-Castro, G.A. Silva, C. Castillo \& Sieverd., presentan esporas ornamentadas con depresiones, pero a diferencia de $A$. minuta son más grandes en diámetro, profundidad y distancia de separación entre ellas (Oehl et al., 2012; Pereira et al., 2015).
Acaulospora papillosa C.M.R. Pereira \& Oehl. Phytotaxa 260(1): 16. 2016. TIPO: BRASIL. Pernambuco, Tamandaré, Reserva Biológica de Saltinho, 843'09"S, $35^{\circ} 10^{\prime} 11^{\prime \prime O}$, bosque tropical húmedo, 01.IV.2015, C. M. R. Pereira 87964 (URM) (isotipos: 87965-87967 (URM), 55192 (ZT)). MycoBank MB 814407. Figs. 2A-E.

Esporas solitarias en el suelo, sin presencia del sáculo esporífero; blancas con tonalidades de amarillo (0/0/40/0), globosas, 62-82 $\mu \mathrm{m}$ de diámetro, al romperse, la pared de la espora se divide en tres grupos, pared externa (OW), intermedia (MW) e interna (IW); la pared externa (OW) con tres capas (OWL1-L3), ninguna de las capas reaccionó al reactivo de Melzer, juntas 3.4 $\mu \mathrm{m}$ de grosor, OWL1 evanescente, hialina, 1.1-2.2 $\mu \mathrm{m}$ de grosor, cubierta de finas papilas, $0.7 \mu \mathrm{m}$ de diámetro, separación entre ellas $1.2 \mu \mathrm{m}$; OWL2 color amarillo claro (0/0/40/0), laminada, lisa, 1.8-2.4 $\mu \mathrm{m}$ de grosor; OWL3 hialina, semiflexible, menor a $1 \mu \mathrm{m}$ de grosor, fuertemente adherida a OWL2; la pared intermedia (MW) con dos capas subhialinas (MWL1-L2), no reaccionan al reactivo de Melzer, semiflexibles, ambas miden $1.2 \mu \mathrm{m}$ de grosor; la pared interna (IW) con dos capas (IWL1-L2) hialinas, semiflexibles; IWL1, menor de $1 \mu \mathrm{m}$, con granulaciones sobre su superficie como la mayoría de las especies de Acaulospora (Oehl et al., 2012); IWL2 $1.5 \mu \mathrm{m}$ de grosor, reaccionó al reactivo de Melzer cambiando su coloración hialina a púrpura rojizo $(20 / 80 / 40 / 0)$ o púrpura intenso (40/80/40/0).

Hábitat y distribución: Acaulospora papillosa solo se había reportado del suelo recuperado de un bosque tropical húmedo en Pernambuco, Brasil. En cultivos puros A. papillosa formó simbiosis micorrízica arbuscular con Zea mays (Pereira et al., 2016). Ahora también se distribuye en el sureste mexicano en el estado de Oaxaca, asociado al suelo de los agaves mezcaleros $A$. angustifolia y A. karwinskii.

Material examinado: MÉXICO. Oaxaca, municipio Ejutla (S2), km 50 carretera Oaxaca - Ejutla, Va- 

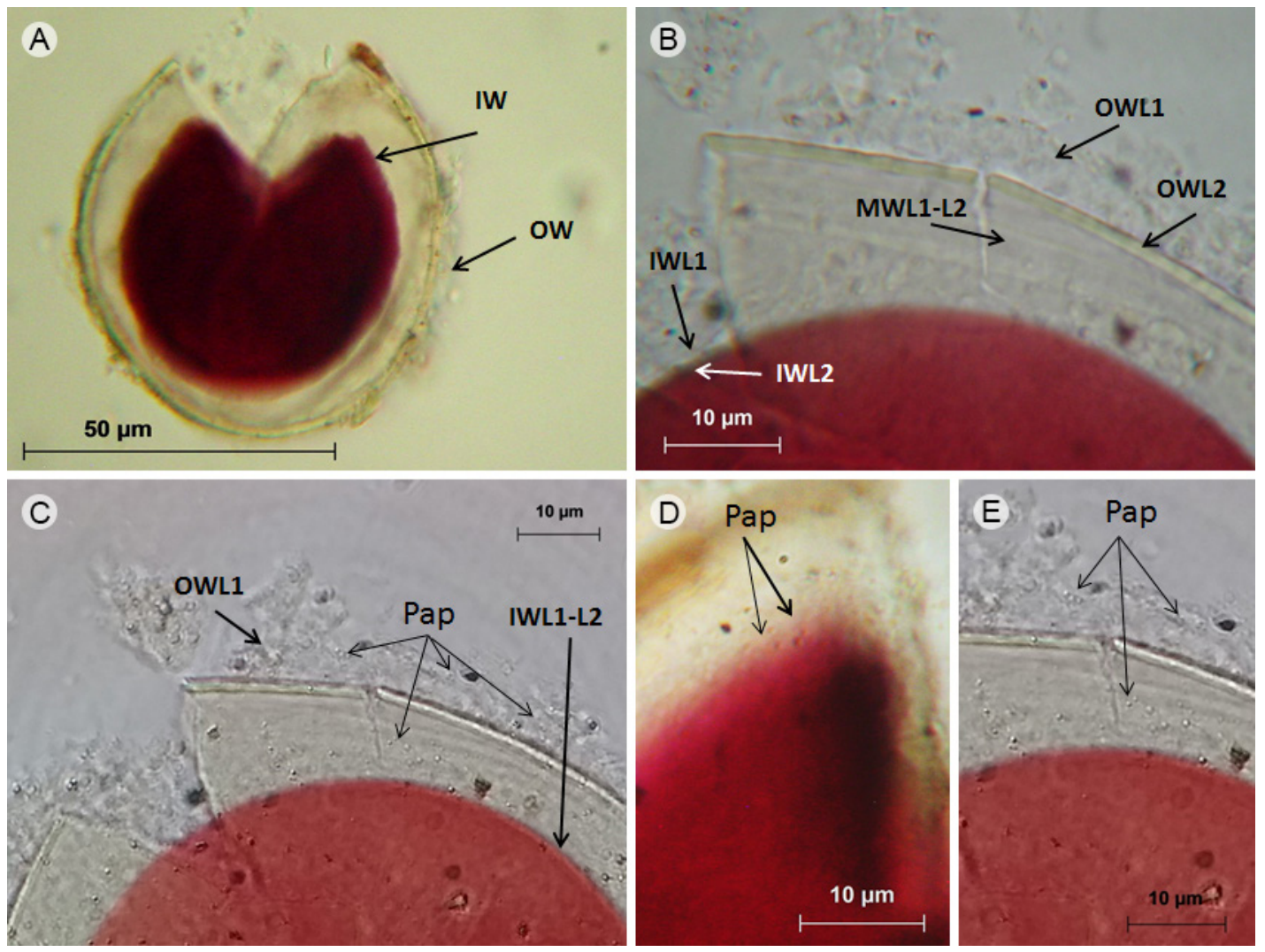

Figura 2: Acaulospora papillosa C.M.R. Pereira \& Oehl. A-E. esporas en reactivo de Melzer, A. espora con pared externa (OW) e interna (IW); B. detalle de la capa evanescente, hialina (OWL1) y laminada (OWL2) en OW; de las capas hialinas intermedias (MWL1-L2) y de las dos capas hialinas internas (IWL1-L2) en donde sólo la capa IWL2 reaccionó al reactivo de Melzer; C. vista de las papilas (Pap) sobre OWL1 y pared interna con dos capas (IWL1-L2); D. papilas (Pap) vistas en primer plano; E. detalle de la ornamentación que consiste de finas papilas (Pap) sobre la capa más externa (OWL1).

lles Centrales de Oaxaca, 1508 m s.n.m., 16³8'31"N, $96^{\circ} 44^{\prime} 04.2^{\prime \prime} \mathrm{O}$, en la rizósfera de $A$. angustifolia (espadín): suelo franco-arenoso (63.4\% arena, $20.2 \%$ limo y $16.4 \%$ arcilla), neutro ( $\mathrm{pH}: 7.19, \mathrm{H}_{2} \mathrm{O}$ ), medianamente rico en MO (2.16\%), muy pobre en fósforo disponible $(0.8 \mathrm{mg}$ $\left.\mathrm{kg}^{-1}\right)$, con altos contenidos de calcio $\left(28\right.$ cmoles $\left._{(+)} \mathrm{kg}^{-1}\right)$, magnesio $\left(6.7\right.$ cmoles $\left._{(+)} \mathrm{kg}^{-1}\right)$ y potasio $\left(0.65\right.$ cmoles $_{(+)}$ $\mathrm{kg}^{-1}$ ) y bajo en sodio $\left(0.18\right.$ cmoles $\left._{(+)} \mathrm{kg}^{-1}\right), 20 . \mathrm{I} .2016, J$. Y. Salmerón-Castro 004 (ENCB). Municipio San Agustín Amatengo (S4), Valles Centrales de Oaxaca, $1387 \mathrm{~m}$ s.n.m., $16^{\circ} 31^{\prime} 05.8^{\prime \prime} \mathrm{N}, 96^{\circ} 47^{\prime} 08.2^{\prime \prime} \mathrm{O}$, en la rizósfera de A. karwinskii (tobasiche): suelo franco-arenoso (63.2\% arena, $18.2 \%$ limo y $18.4 \%$ arcilla), ligeramente alcalino (pH: 7.86, $\left.\mathrm{H}_{2} \mathrm{O}\right)$, medianamente rico en $\mathrm{MO}(2.51 \%)$ y muy pobre en fósforo disponible $\left(0.4 \mathrm{mg} \mathrm{kg}^{-1}\right)$, 20.I.2016,
J. Y. Salmerón-Castro 006 (UAMIZ), 007 (UAMIZ). Municipio San Luis Amatlán, Miahuatlán (S6), Sierra Sur de Oaxaca, 1525 m s.n.m., 16²2'28.7"N y 96³9'11.1"O, en la rizosfera de $A$. karwinskii (madrecuishe): suelo francoarenoso (57.6\% arena, $26 \%$ limo y $16.4 \%$ arcilla), ligeramente alcalino $\left(\mathrm{pH}: 8.02, \mathrm{H}_{2} \mathrm{O}\right)$, rico en $\mathrm{MO}: 5.72 \%$, muy pobre en fósforo disponible $\left(1.2 \mathrm{mg} \mathrm{kg}^{-1}\right)$; con altos contenidos de calcio $\left(28.5\right.$ cmoles $\left._{(+)} \mathrm{kg}^{-1}\right)$ y magnesio (7.9 cmoles $\left._{(+)} \mathrm{kg}^{-1}\right)$, mediano en potasio $\left(0.43\right.$ cmoles $\left._{(+)} \mathrm{kg}^{-1}\right) \mathrm{y}$ bajo en sodio $\left(0.13\right.$ cmoles $\left._{(+)} \mathrm{kg}^{-1}\right)$, 21.I.2016, J. Y. Salmerón-Castro 016 (UAMIZ).

Comentarios taxonómicos: Acaulospora papillosa puede ser reconocida por las pequeñas papilas en la capa evanescente (OWL1) de la pared de las esporas, ya que 
en la mayoría de las especies de Acaulospora con ornamentaciones como espinas ( $A$. spinosa), tubérculos $(A$. tuberculata), depresiones (A. scrobiculata), verrugas $(A$. ignota), pliegues cerebriformes (A. rehmii) o doble ornamentación ( $A$. bireticulata o $A$. reducta) se presentan en la pared laminada (OWL2) (Oehl et al., 2012; Schüßler, 2017). Las únicas especies de Acaulospora con una capa externa (OWL1) con ornamentaciones son A. pustulata, A. tortuosa (Palenzuela et al., 2013) y A. colliculosa (Kaonongbua et al., 2010). Las dos primeras especies al presentar una ornamentación de pústulas o estructuras como hifas irregulares muy grandes difieren de las diminutas papilas presentes en A. papillosa; mientras que A. colliculosa además de presentar protuberancias hemisféricas mayores a $1 \mu \mathrm{m}$ de diámetro, presenta dos cicatrices que revelan su formación entrofosporoide y no acaulosporoide como en A. papillosa. Acaulospora papillosa puede ser confundida con A. morrowiae Spain \& N.C. Schenck o $A$. rugosa J.B. Morton, por su similitud en el tamaño de las esporas; sin embargo, ninguna de estas especies presenta ornamentación en la capa evanescente como A. papillosa.

Acaulospora reducta Oehl, B.T. Goto \& C.M.R. Pereira, Mycotaxon 130(4): 986. 2016. TIPO: BRASIL. Pernambuco, Goiana, Estación experimental de Itapirema, 13 m s.n.m., 7³8'20"S, 3457'10"O, plantaciones agrícolas con Manihot esculenta Crantz, 1.VI.2011, C. M. R. Pereira s.n. (holotipo: 87697 (URM), isotipos: 87698 (URM), 55894 (ZT)). MycoBank 812332. Figs. 3A-F.

Esporas libres en el suelo y no en esporocarpos, cicatriz en forma de estrella irregular, $14.5 \mu \mathrm{m}$ de ancho, $17.8 \mu \mathrm{m}$ de largo; restos del sáculo esporífero color amarillo claro (0/10/40/0), esporas color anaranjado (0/20/60/0), $150 \mu \mathrm{m}$ de diámetro, al romperse la pared de las esporas se divide en tres grupos, pared externa (OW), intermedia (MW) e interna (IW); la pared más externa (OW) con tres capas (OWL1-L3), ninguna de estas capas reaccionó al reactivo de Melzer; OWL1 hialina, semipermanente, de $2 \mu \mathrm{m}$ de grosor; OWL2 color anaranjado (0/20/60/0), laminada, 5.8-9.5 $\mu \mathrm{m}$ de grosor, con grandes depresiones, algunas circulares, $5.9 \mu \mathrm{m}$ de diámetro, otras subcirculares, $7.8 \mu \mathrm{m}$ de largo, $5.2 \mu \mathrm{m}$ de ancho, la mayoría de forma irregular, hasta $7.8 \mu \mathrm{m}$ de ancho, $17.9 \mu \mathrm{m}$ de largo, 2-4 $\mu \mathrm{m}$ de profundidad, las depresiones de textura rugosa interna con pequeñas depresiones, menos de $1 \mu \mathrm{m}$ de diámetro; OWL3 similar en color a OWL2, menor a $1 \mu \mathrm{m}$ de grosor; la pared intermedia (MW) con dos capas hialinas (MWL1-L2), que no reaccionaron al reactivo de Melzer, semiflexibles, fuertemente adheridas entre ellas, casi $2 \mu \mathrm{m}$ de grosor; la pared interna (IW) con al menos dos capas (IWL1-L2) hialinas, semiflexibles; IWL1 $1.6 \mu \mathrm{m}$ de grosor con excrecencias sobre su superficie; IWL2 $3.1 \mu \mathrm{m}$ de grosor con reacción al reactivo de Melzer tiñéndose hacia una coloración púrpura-rosado (0/60/30/10). De acuerdo a Pereira et al. (2015), la pared interna IW de $A$. reducta presenta tres capas; sin embargo, no fue posible distinguir la tercera capa IWL3 en nuestra revisión, probablemente por su delgadez y por estar fuertemente adherida a IWL2.

Hábitat y distribución: Acaulospora reducta solo se había reportado del suelo recuperado de bosque tropical húmedo, de regiones semiáridas en la "Caatinga" y de una sabana en "El Cerrado" del trópico brasileño (Pereira et al., 2015). Ahora también se distribuye en el sureste mexicano en el estado de Oaxaca, asociado a la rizósfera de Agave karwinskii.

Material examinado: MÉXICO. Oaxaca, municipio San Agustín Amatengo (S3), Valles Centrales de Oaxaca, 1387 m s.n.m., $16^{\circ} 31^{\prime} 05.8^{\prime \prime} \mathrm{N}$ y 96 $6^{\circ} 47^{\prime} 08.2^{\prime \prime O}$, en suelo rizosférico de Agave karwinskii (san martín): suelo arenoso-franco (79.2\% arena, $16.4 \%$ limo y $4.4 \%$ arcilla), neutro ( $\mathrm{pH}: 7.28, \mathrm{H}_{2} \mathrm{O}$ ), medianamente rico en materia orgánica (3.26\%), muy pobre en fósforo disponible $(0.7$ $\left.\mathrm{mg} \mathrm{kg}^{-1}\right)$; con altos contenidos de calcio $\left(15\right.$ cmoles $\left._{(+)} \mathrm{kg}^{-1}\right)$ y magnesio $\left(7.5\right.$ cmoles $\left._{(+)} \mathrm{kg}^{-1}\right)$ y bajos en potasio $(0.29$ cmoles $\left._{(+)} \mathrm{kg}^{-1}\right)$ y sodio $\left(0.14\right.$ cmoles $\left._{(+)} \mathrm{kg}^{-1}\right)$, 20.I.2016, J. Y. Salmerón-Castro 008b (ENCB).

Comentarios taxonómicos: Acaulospora reducta, al presentar esporas con una pared laminada (OWL2) con 

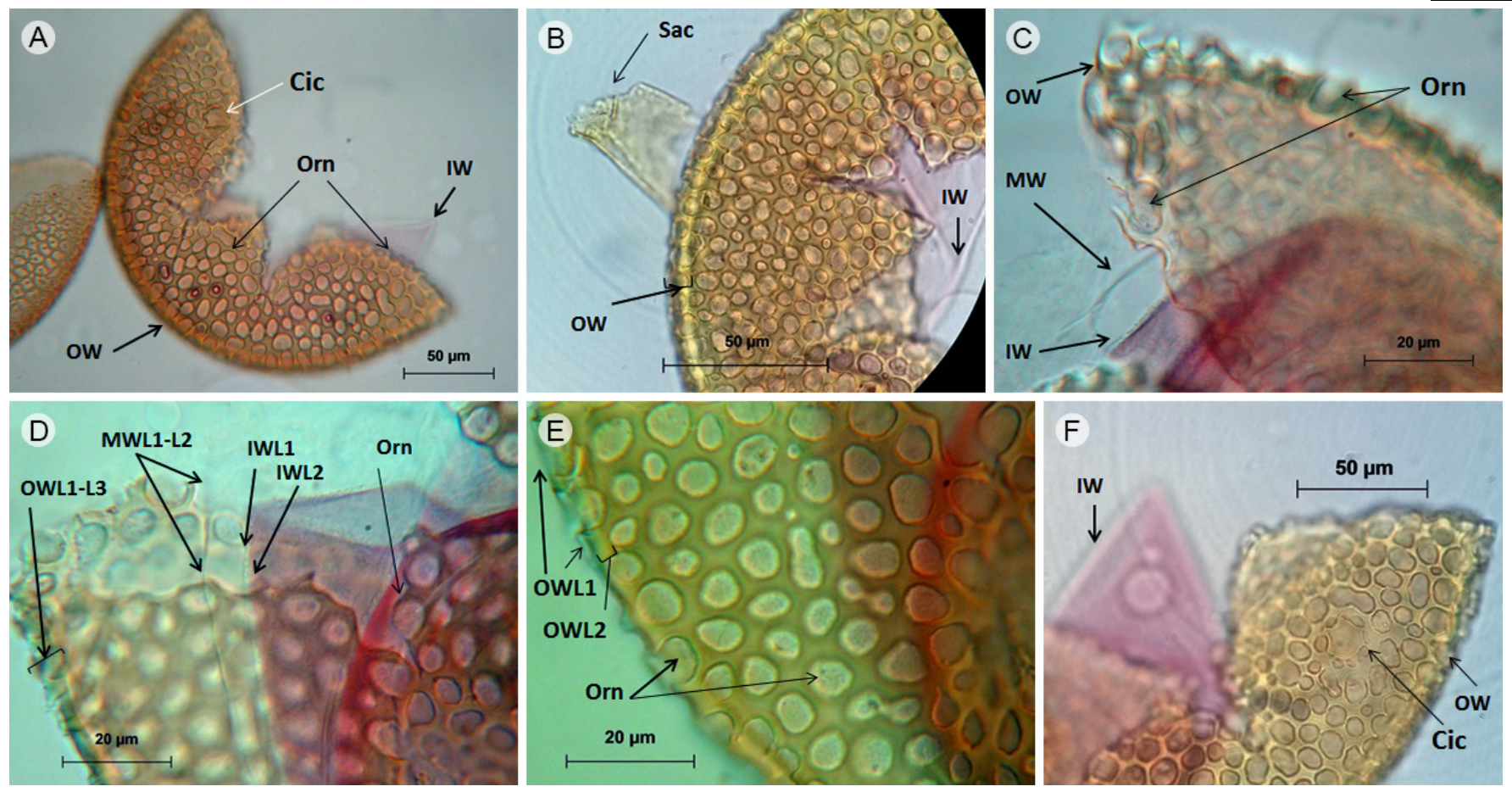

Figura 3: Acaulospora reducta Oehl, B.T. Goto \& C.M.R. Pereira. A-F. esporas en reactivo de Melzer (solo la segunda capa interna (IWL2) hialina se tiñe púrpura-rosado (0/60/30/10) en el reactivo de Melzer), A. detalle de la ornamentación (Orn), cicatriz (Cic), pared externa (OW) e interna (IW) de la espora; B. espora con restos del sáculo (Sac), pared externa (OW) e interna (IW); C. espora con la pared externa (OW), intermedia (MW) e interna (IW) y detalle de la ornamentación (Orn); D. número de capas (L) en la pared externa (OWL1-L3), intermedia (MWL1-L2) e interna (IWL1-L2) con detalle de la ornamentación (Orn) en OW; E. espora con la capa hialina más externa (OWL1) y la capa laminada (OWL2) que muestra la ornamentación (Orn) consistente de grandes depresiones y en su interior tienen otras depresiones pequeñas; F. espora con cicatriz (Cic) en la pared externa $(\mathrm{OW})$ y reacción del reactivo de Melzer en la pared interna (IW).

doble ornamentación consistente de grandes depresiones irregulares $(7.8-5.2 \times 7.8-17.9 \mu \mathrm{m})$ y una rugosidad que consiste de pequeñas depresiones en su interior $(<1 \mu \mathrm{m})$ puede ser fácilmente diferenciada de otras especies del género Acaulospora (Oehl et al., 2012). Solo Acaulospora bireticulata, A. elegans Trappe \& Gerd. y A. denticulata Sieverd. \& Toro (Oehl et al., 2012) tienen doble ornamentación; sin embargo, estas especies no presentan depresiones en la capa laminada como $A$. reducta, sino proyecciones poligonales redondeados en la punta y encerrados por un retículo ( $A$. bireticulata y $A$. elegans) o sin este retículo (A. denticulata). Acaulospora reducta puede confundirse con $A$. excavata y $A$. verna ya que estas especies también presentan esporas ornamentadas con grandes depresiones en la capa laminada; sin embargo, el análisis detallado de las ornamentaciones permite diferenciarlas. En $A$. excavata las depresiones son más circulares y homogéneas en cuanto a su diámetro y profundidad, además de que la superficie de los hoyos es lisa, mientras que en $A$. reducta las depresiones son más irregulares y están ornamentadas en su interior. Por último, Acaulospora reducta difiere de $A$. verna en el tamaño promedio de las esporas $(150 \mu \mathrm{m}$ vs $75 \mu \mathrm{m}$ ) y en que la superficie interna de las depresiones está ornamentada y no es lisa como en A. verna (Pereira et al., 2015).

Paraglomus bolivianum (Sieverd. \& Oehl) Oehl \& G.A. Silva, J. Appl. Bot. Food. Quality. 86: 115. 2013. = Pacispora boliviana Sieverd. \& Oehl, J. Appl. Bot. 78: 79. 2004. TIPO: BOLIVIA. Santa Cruz de la Sierra, 
Gran Chaco, 550 m s.n.m., $18^{\circ} 05^{\prime} \mathrm{S} ; 6^{\circ} 20^{\prime} \mathrm{O}$, pastizal degradado en un ecosistema semiárido, s.f., s.c. (Nr. 35-3501 (ZT)). MycoBank 488665. Figs. 4A-D.

Esporocarpos desconocidos; esporas solitarias en el suelo, color pardo claro (0/20/80/0), globosas, $85-90$ $\mu \mathrm{m}$ de diámetro; al romperse la pared de la espora se separa en dos grupos, pared externa (OW) e interna (IW); la pared externa con tres capas (OWL1-OWL3) que no reaccionan al reactivo de Melzer; OWL1 hialina, semipermanente, lisa, $2.8 \mu \mathrm{m}$ de grosor, ausente en esporas maduras; OWL2 color pardo claro $(0 / 20 / 80 / 0)$, permanente, laminada, $3.1 \mu \mathrm{m}$ de grosor, ornamentada con depresiones, $3.6 \mu \mathrm{m}$ de diámetro, $1.5 \mu \mathrm{m}$ de profundidad, separadas $2.5 \mu \mathrm{m}$ en promedio; OWL3 pardo muy cla- ro $(0 / 0 / 20 / 0)$, permanente, lisa, menor a $1 \mu \mathrm{m}$ de grosor, fuertemente adherida a OWL2; la pared interna (IW) con tres capas (IWL1-L3) hialinas que no reaccionan al reactivo de Melzer, juntas $3.1 \mu \mathrm{m}$ de grosor; IWL1 menor a 0.7 $\mu \mathrm{m}$; IWL2 $1.9 \mu \mathrm{m}$ y IWL3 menor a $0.5 \mu \mathrm{m}$ de grosor; hifa de sostén sub-hialina, recta, $5.9 \mu \mathrm{m}$ de amplitud en la base de la espora, con dos capas (HWL1-L2) continuas con la pared externa de la espora, juntas $2.2 \mu \mathrm{m}$ de grosor, poro de la hifa de sostén cerrado por un septo que se origina de la capa OWL3.

Hábitat y distribución: Paraglomus bolivianum solo se había reportado de Suramérica, de Santa Cruz, Bolivia, en un pastizal (pH: 6.5) y de Pernambuco, Brasil, en bosque seco "Caatinga" (pH: 5.2-6.8) y vegetación coste-
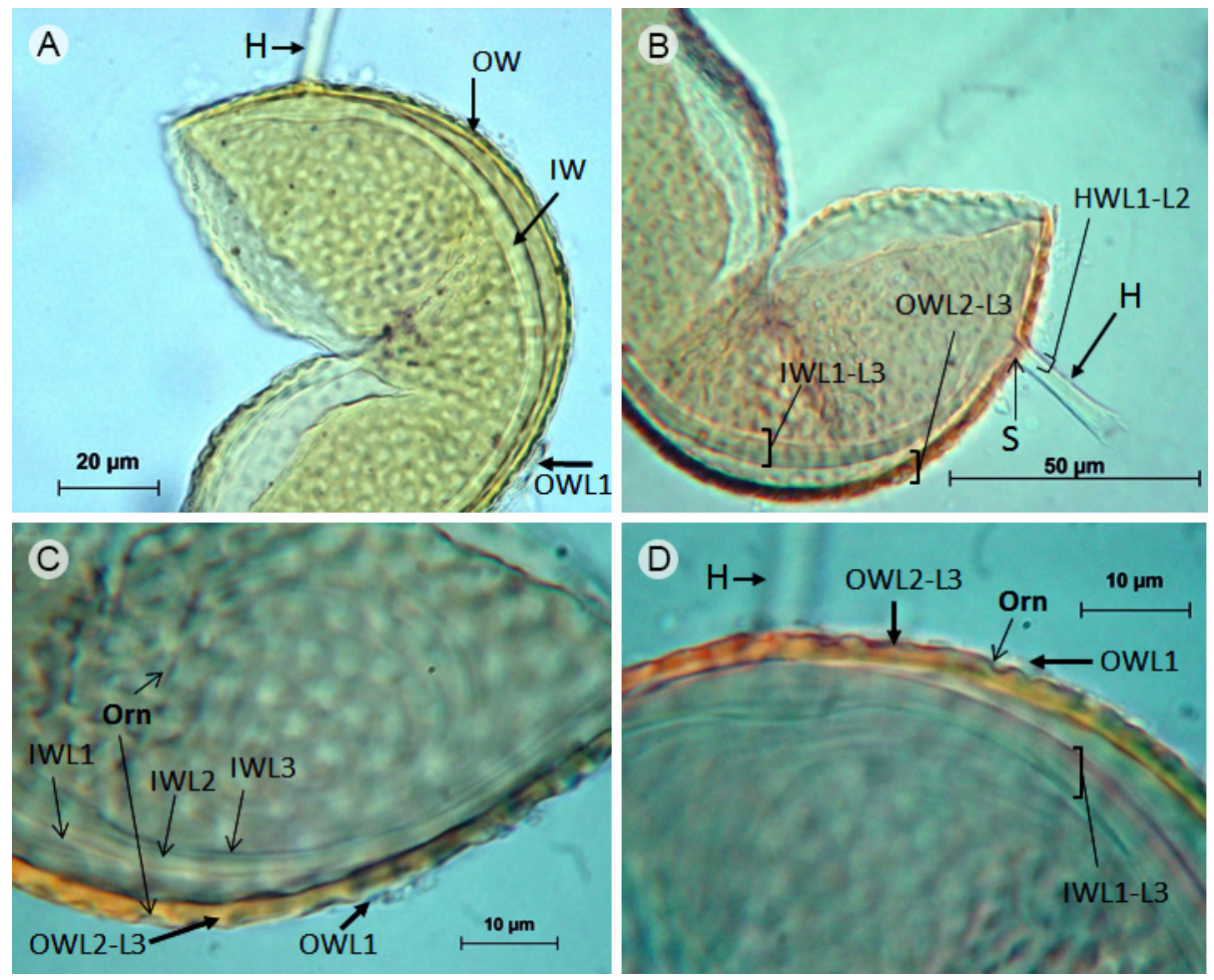

Figura 4: Paraglomus bolivianum (Sieverd. \& Oehl) Oehl \& G.A. Silva = Pacispora boliviana Sieverd. \& Oehl. A-D. esporas en reactivo de Melzer sin reacción, A. espora con hifa $(\mathrm{H})$ y dos grupos de paredes, una externa (OW) y una interna (IW), en OW se aprecian restos de la capa hialina más externa (OWL1); B. pared externa con una capa laminada (OWL2) y una permanente (OWL3), pared interna constituida por tres capas hialinas (IWL1-L3) y detalle de la hifa (H) con un septo (S) y dos capas (HWL1-L2) que son continuas con la pared externa de la espora; C. capa hialina (OWL1), laminada OWL2 y capa permanente OWL3 en la pared externa de la espora, nótese la ornamentación (Orn) en la capa laminada (OWL2) consistente de grandes depresiones, además detalle de las tres capas (IWL1, IWL2 y IWL3) hialinas en la pared interna de la espora; D. espora con hifa (H) presentando la capa hialina (OWL1), laminada (OWL2) y permanente (OWL3) en la pared externa de la espora y detalle de las tres capas hialinas (IWL1-L3) en la pared interna de la espora. 
ra ((pH: 5.1) (Oehl y Sieverding, 2004; Aragão de Mello et al., 2013)). Ahora se distribuye también en el sureste mexicano en el estado de Oaxaca, asociado a la rizósfera de Agave angustifolia y A. karwinskii.

Material examinado: MÉXICO. Oaxaca, municipio San Pedro Totolapan (S1), Valles Centrales de Oaxaca, 940 m s.n.m., $16^{\circ} 40^{\prime} 58^{\prime \prime} \mathrm{N}, 96^{\circ} 18^{\prime} 23^{\prime \prime} \mathrm{O}$, en suelo rizosférico de A. karwinskii (cuishe): suelo franco-arenoso (66\% arena, $27.6 \%$ limo y $6.4 \%$ arcilla), neutro $\left(\mathrm{pH}: 7.52, \mathrm{H}_{2} \mathrm{O}\right)$, medianamente rico en materia orgánica $(2.68 \%)$, muy pobre en fósforo disponible $\left(4 \mathrm{mg} \mathrm{kg}^{-1}\right)$; con altos contenidos de calcio $\left(21.2\right.$ cmoles $\left._{(+)} \mathrm{kg}^{-1}\right)$ y magnesio $\left(16.5\right.$ cmoles $\left._{(+)} \mathrm{kg}^{-1}\right)$ $\mathrm{y}$ bajos en potasio $\left(0.45\right.$ cmoles $\left._{(+)} \mathrm{kg}^{-1}\right)$ y sodio $(0.18 \mathrm{cmo}-$ les $\left._{(+)} \mathrm{kg}^{-1}\right), 20 . I .2016$, J. Y. Salmerón-Castro 002 (ENCB). Municipio Ejutla (S2), km 50 carretera Oaxaca - Ejutla, Valles Centrales de Oaxaca, 1508 m s.n.m., 16³8'31"N, $96^{\circ} 44^{\prime} 04.2^{\prime \prime} \mathrm{O}$, en la rizósfera de $A$. angustifolia: suelo franco-arenoso (63.4\% arena, $20.2 \%$ limo y $16.4 \%$ arci1la), neutro ( $\mathrm{pH}: 7.19, \mathrm{H}_{2} \mathrm{O}$ ), medianamente rico en $\mathrm{MO}$ $(2.16 \%)$, muy pobre en fósforo disponible $\left(0.8 \mathrm{mg} \mathrm{kg}^{-1}\right)$, con altos contenidos de calcio $\left(28\right.$ cmoles $\left._{(+)} \mathrm{kg}^{-1}\right)$, magnesio $\left(6.7\right.$ cmoles $\left._{(+)} \mathrm{kg}^{-1}\right)$ y potasio $\left(0.65 \mathrm{cmoles}_{(+)} \mathrm{kg}^{-1}\right)$ y bajo en sodio $\left(0.18\right.$ cmoles $\left._{(+)} \mathrm{kg}^{-1}\right), 20 . I .2016, J$. Y. SalmerónCastro 003 (UAMIZ), 006 (UAMIZ).

Comentarios taxonómicos: Paraglomus bolivianum es la única especie conocida de Paraglomerales que presenta esporas pigmentadas y una ornamentación con depresiones en la capa OWL2 muy grandes y profundas (en promedio $3.6 \mu \mathrm{m}$ de diámetro, $1.5 \mu \mathrm{m}$ de profundidad y una separación de $2.5 \mu \mathrm{m}$ ) en comparación con $P$. pernambucanum Oehl, C.M. Mello, Magna \& G.A. Silva que tiene esporas hialinas o ligeramente amarillas y una capa laminada con depresiones más pequeñas $(0.5-$ $1.1 \mu \mathrm{m}$ de diámetro, $0.5-1.0 \mu \mathrm{m}$ de profundidad y 1.3$2.4 \mu \mathrm{m}$ de separación entre ellas) y superficiales (Aragão de Mello et al., 2013). Paraglomus brasilianum (Spain \& J. Miranda) Morton \& Redecker es otra especie del género que presenta una capa OWL2 con ornamentación pero con un patrón finamente reticulado (INVAM, 2017) y no en depresiones como en $P$. bolivianum o $P$. pernambucanum. En el caso de P. occultum (C. Walker) J.B. Morton \& D. Redecker, P. laccatum (Blaszk.) Renker, Blaszk. \& Buscot, P. majewskii Blaszk. \& Kovács y $P$. turpe Oehl, V.M. Santos \& Palenz. estos HMA forman esporas hialinas a subhialinas y sin ninguna ornamentación en la capa laminada OWL2, así que pueden ser fácilmente diferenciadas de las esporas pardo claro y ornamentadas de $P$. bolivianum (Blaszkowski et al., 2012; Oehl et al., 2016; INVAM, 2017).

Paraglomus bolivianum fue descrito originalmente como Pacispora boliviana Sieverd. \& Oehl debido a que todas las especies que conforman el género Pacispora Sieverd. \& Oehl presentan esporas tipo glomoide con una pared compuesta de dos grupos; cada grupo está constituido por tres capas y por lo regular la segunda capa interna (IWL2) reacciona al reactivo de Melzer (Oehl y Sieverding, 2004). De esta manera, $P$. bolivianum se puede diferenciar de Pacispora franciscana Sieverd. \& Oehl y P. robigina Sieverd. \& Oehl porque son especies de HMA con esporas sin ornamentaciones en la capa OWL2, y la capa IWL2 reacciona al reactivo de Melzer. Asimismo, $P$. bolivianum, al presentar esporas con una capa laminada con grandes depresiones y sin reacción al reactivo de Melzer en ninguna de sus capas (Figs. 4A-D), se puede diferenciar de Pacispora chimonobambusae (C.G. Wu \& Y.S. Liu) Sieverd. \& Ochl ex C. Walker, Vestberg \& Schuessler, P. dominikii Sieverd. \& Oehl, P. scintillans (S.L. Rose \& trappe) Sieverd. \& Oehl ex C. Walker, Vestberg \& Schuessler, P. coralloidea Sieverd. \& Oehl y $P$. patagonica (Novas \& Fracchia) C. Walker, Vestberg \& Schuessler porque en estas especies las esporas presentan una capa OWL2 con una ornamentación consistente de proyecciones semejantes a tubos o verrugas y la capa IWL2 tiene reacción al reactivo de Melzer (Oehl y Sieverding, 2004; Novas et al., 2005).

\section{DISCUSIÓN}

Esta investigación indicó que en el suelo rizosférico de Agave angustifolia y de $A$. karwinskii de los Valles Cen- 
trales y Sierra Sur de Oaxaca, México, hay una riqueza de especies aún no conocida como ocurrió con Acaulospora minuta (Fig. 1), A. papillosa (Fig. 2), A. reducta (Fig. 3) y Paraglomus bolivianum (Fig. 4) que solo se habían registrado en África (A. minuta) o Suramérica (Bolivia y Brasil) (Oehl y Sieverding, 2004; Oehl et al., 2011; Aragão de Mello et al., 2013; Pereira et al., 2015, 2016). Lo anterior sugería que tenían una distribución restringida. Estas especies de HMA representan cuatro nuevos registros para México, puesto que no habían sido reportadas con anterioridad en ecosistemas naturales ni en agro-ecosistemas mexicanos (Violi et al., 2008; Alarcón et al., 2012; Méndez-Cortés et al., 2012; Montaño et al., 2012; Chimal-Sánchez et al., 2015, 2016; Posada et al., 2016; Álvarez et al., 2017; Carballar-Hernández et al., 2017; Varela-Fregoso et al., 2017); sobre todo en agaves mezcaleros de Oaxaca (Carballar-Hernández, 2009; Carballar-Hernández et al., 2013) o de otros estados del país (Ochoa-Meza et al., 2009; Trinidad-Cruz et al., 2017). Así, en México ahora se contabilizan 148 especies de hongos formadores de micorriza arbuscular, riqueza que representa casi $50 \%$ de los HMA formalmente descritos mundialmente (Schüßler, 2017). Es conveniente señalar que en México aún son escasos los trabajos que cuentan con una descripción taxonómica como la presentada en este estudio, así como respaldos de herbario que permitan estudios posteriores relacionados con su diversidad, distribución, taxonomía, tipos de hospederos y condiciones edáficas específicas para su desarrollo, entre otras. Finalmente, la interacción de los HMA con los agaves que se usan en la producción del mezcal, como A. angustifolia y A. karwinskii, puede ser de gran utilidad para preservar, propagar y conservar este recurso vegetal de lento crecimiento, establecidos en suelos con regímenes hídricos secos y baja disponibilidad de fósforo (Cuadro 1), sumado a que los agaves están amenazados por su comercialización y explotación de los ecosistemas naturales sin un plan de manejo a corto y largo plazo; por lo que el uso de inoculantes micorrízicos podría ser una opción viable en la producción de agaves mezcaleros.

\section{CONTRIBUCIÓN DE AUTORES}

NVP e IRJ conformaron la idea y el diseño experimental. Todos los autores, a excepción de LVF, salieron a campo para la recolecta de las muestras de suelo. JYSC y ECHS extrajeron las esporas de los HMA del suelo. IRJ y JYSC establecieron las macetas de propagación e hicieron los análisis del suelo. ECHS y LVF realizaron la determinación taxonómica de los HMA y tomaron las fotografías. ECHS ilustró las leyendas de las esporas de HMA con la correspondiente nomenclatura. ECHS e IRJ escribieron el artículo en donde todos los autores contribuyeron a la conformación de resultados, discusión, revisión y aprobación del manuscrito final.

\section{AgradeCIMIENTOS}

A Genaro Sierra de Ejutla, Misael Ramírez de Tlacolula, a Celina y Miguel Pérez de Miahuatlán por su apoyo en la recolecta en campo. Así como a Rosalva García Sánchez (FES-UNAM), Aniceto Mendoza Ruiz y Blanca Pérez García (UAM-IZ) por su apoyo con el equipo de microscopía con que se obtuvieron las microfotografías de los HMA. A los revisores y editora Marie-Stéphanie Samain que, con sus comentarios y correcciones acertadas, ayudaron a mejorar la información presentada en el presente artículo.

\section{LITERATURA CITADA}

Alarcón, A., L. V. Hernández-Cuevas, R. Ferrera-Cerrato y A. Franco-Ramírez. 2012. Diversity and applications of arbuscular mycorrhizal fungi in Mexico. Journal of Biofertilizers and Biopesticides 3(1): 115. DOI: https:// dx.doi.org/10.4172/2155-6202.1000115

Álvarez-Sánchez, F. J., I. Sánchez-Gallen, L. V. HernándezCuevas, L. Hernández-Oro y P. Meli. 2017. Diversidad, abundancia y variación estacional en la comunidad de hongos micorrizógenos arbusculares en la selva Lacandona, Chiapas, México. Scientia Fungorum 45: 37-51.

Aragão de Mello, C. M., G. Alves da Silva, D. M. Azevedo de Assis, J. Souza de Pontes, A. C. de Almeida Ferreira, M. P. Carneiro Leão, H. E. Evangelista Vieira, L. Costa Maia y F. Oehl. 2013. Paraglomus pernambucanum sp. nov. and 
Paraglomus bolivianum comb. nov., and biogeographic distribution of Paraglomus and Pacispora. Journal of Applied Botany and Food Quality 86: 113-125.

Blaszkowski, J. 2017. Taxonomy of arbuscular fungi. http:// www.zor.zut.edu.pl/Glomeromycota/Taxonomy.html (consultado diciembre de 2017).

Błaszkowski, J., G. M. Kovács, B. K. Gáspár, T. K. Balázs, F. Buscot y P. Ryszka. 2012. The arbuscular mycorrhizal Paraglomus majewskii sp. nov. represents a distinct basal lineage in Glomeromycota. Mycologia 104(1): 148-156. DOI: https://dx.doi.org/10.3852/10-430

Camargo-Ricalde, S. L., S. S. Dhillion y C. Jiménez-González. 2003. Mycorrhizal perennials of the "matorral xerófilo" and the "selva baja caducifolia" communities in the semiarid Tehuacán-Cuicatlán Valley, Mexico. Mycorrhiza 13(2): 77-83. DOI: https://dx.doi.org/10.1007/s00572002-0203-8

Carballar-Hernández, S. 2009. Variación temporal de la diversidad de hongos de micorriza arbuscular y el potencial micorrízico en especies silvestres de Agave en Oaxaca. Tesis de maestría. Centro Interdisciplinario de Investigación para el Desarrollo Integral Regional (CIIDIR), Instituto Politécnico Nacional (IPN). Oaxaca, México. $72 \mathrm{pp}$.

Carballar-Hernández, S., F. J. Palma-Cruz, L. V. HernándezCuevas y C. Robles. 2013. Arbuscular mycorrhizal potential and mycorrhizal fungi diversity associated with Agave potatorum Zucc. in Oaxaca, Mexico. Ecological Research 28(2): 217-226. DOI: https://dx.doi. org/10.1007/s11284-012-1008-7

Carballar-Hernández, S., L. V. Hernández-Cuevas, N. M. Montaño, J. Larsen, R. Ferrera-Cerrato, O. R. TaboadaGaytán, A. M. Montiel-González y A. Alarcón. 2017. Native communities of arbuscular mycorrhizal fungi associated with Capsicum annuum L. respond to soil properties and agronomic management under field conditions. Agriculture, Ecosystems and Environment 245: 43-51. DOI: https://dx.doi.org/10.1016/j. agee.2017.05.004

Chimal-Sánchez, E., R. García-Sánchez y L. V. HernándezCuevas. 2015. Gran riqueza de hongos micorrizógenos arbusculares en el Valle del Mezquital, Hidalgo, México. Revista Mexicana de Micología 41: 15-26.

Chimal-Sánchez, E., N. M. Montaño, S. L. Camargo-Ricalde, R. García-Sánchez y L. V. Hernández-Cuevas. 2016. Nuevos registros de hongos micorrizógenos arbusculares para México. Revista Mexicana de Biodiversidad 87(1): 242-247. DOI: https://doi.org/10.1016/j.rmb.2016.01.024 García-Mendoza, A. J., M. J. Ordoñez y M. Briones-Salas. 2004. Biodiversidad de Oaxaca. Universidad Nacional Autónoma de México (UNAM), Fondo Oaxaqueño para la Conservación de la Naturaleza (FOCN) y World Wildlife Fund (WWF). Cd. Mx., México. 609 pp.

García-Sánchez, R., A. Monroy-Ata y E. Chimal-Sánchez. 2008. Hongos micorrizógenos arbusculares asociados a diferentes plantas y matorrales del Valle del Mezquital, Hidalgo, México. In: Montaño, N. M., S. L. CamargoRicalde, R. García-Sánchez y A. Monroy-Ata (eds.). Micorrizas arbusculares en ecosistemas áridos $\mathrm{y}$ semiáridos. Mundi-Prensa, S.A. de C.V., Instituto Nacional de Ecología (INE), Secretaría de Medio Ambiente y Recursos Naturales (SEMARNAT), Universidad Autónoma Metropolitana-Iztapalapa (UAM-IZ)-Facultad de Estudios Superiores-Zaragoza-Universidad Nacional Autónoma de México (FES-UNAM). Cd. Mx., México. Pp. 123-136.

Gerdemann, W. J. y H. T. Nicolson. 1963. Spores of mycorrhizal Endogone species extracted from soil by wet sieving and decanting. Transactions of the British Mycological Society 46: 235-244. DOI: https://doi.org/10.1016/ S0007-1536(63)80079-0

Hernández-Morales, J. L., C. López-Sánchez y F. J. PalmaCruz. 2014. Caracterización morfológica de micorriza arbuscular asociada a Agave potatorum Zucc. con potencial de uso agronómico. Revista Mexicana de Agroecosistemas 1(2): 82-93.

Holmgren, P. K., H. N. Holmgren y C. L. Barnett 1990. Index herbarorium. Part I. The herbaria of the world. $8^{\mathrm{a}}$ ed. International Association for Plant Taxonomy and New York Botanical Garden. New York, USA.

INEGI. 2010. Compendios de información geográfica municipal de los Estados Unidos Mexicanos: Oaxaca. 
Instituto Nacional de Estadística, Geografía e Informática. Aguascalientes, México.

INVAM. 2017. Species descriptions from reference cultures. International Culture Collection of (Vesicular) Arbuscular Mycorrhizal Fungi (INVAM). https://invam.wvu.edu/ (consultado diciembre de 2017).

Jackson, L. M. 1976. Análisis químico de suelos. Ed. Omega. Barcelona, España. 662 pp.

Kaonongbua, W., J. B. Morton y J. D. Bever. 2010. Taxonomic revision transferring species in Kuklospora to Acaulospora (Glomeromycota) and a description of Acaulospora colliculosa sp. nov. from field collected spores. Mycologia 102(6): 1497-1509. DOI: https:// dx.doi.org/10.3852/10-011

Méndez Cortés, H., J. G. Marmolejo Monsivais, V. Olalde Portugal, C. M. Cantú Ayala y L. Varela Fregoso. 2012. Nuevos registros de hongos micorrizógenos arbusculares para México. Revista Mexicana de Micología 36: 49-56.

Montaño, N. M., S. L. Camargo-Ricalde, R. García-Sánchez y A. Monroy-Ata. 2008. Micorrizas arbusculares en ecosistemas áridos y semiáridos. Mundi-Prensa, S.A de C.V., Instituto Nacional de Ecología (INE), Secretaría de Medio Ambiente y Recursos Naturales (SEMARNAT), Universidad Autónoma Metropolitana-Iztapalapa(UAMIZ), Facultad de Estudios Superiores-ZaragozaUniversidad Nacional Autónoma de México (FESUNAM). Cd. Mx., México. Pp. 266.

Montaño, N. M., A. Alarcón, S. L. Camargo-Ricalde, L. V. Hernández-Cuevas, J. Álvarez-Sánchez, M. C. GonzálezChávez, M. Gavito, I. Sánchez-Gallen, J. Ramos-Zapata, P. Guadarrama, I. E. Maldonado-Mendoza, S. CastilloArgüero, R. García-Sánchez, D. Trejo y R. FerreraCerrato. 2012. Research on arbuscular mycorrhizae in Mexico: an historical synthesis and future prospects. Symbiosis 57(3): 111-126. DOI: https://doi.org/10.1007/ s13199-012-0184-0

Novas, V. M., S. Fracchia, A. Menéndez, D. Cabral y A. Godeas. 2005. Glomus patagonicum sp. nov. (Glomerales), a new arbuscular mycorrhizal fungus from Argentina. Nova Hedwigia 80(3): 533-540. DOI: https://doi. org/10.1127/0029-5035/2005/0080-0533
Ochoa-Meza, A., M. Esqueda, R. Fernández-Valle y R. Herrera-Peraza. 2009. Variación estacional de hongos micorrízicos arbusculares asociados con Agave angustifolia Haw. en la Sierra Sonorense, México. Revista Fitotecnia Mexicana 32(3): 189-199.

Oehl, F. y E. Sieverding. 2004. Pacispora, a new vesicular arbuscular mycorrhizal fungal genus in the Glomeromycetes. Journal of Applied Botany 78: 72-82.

Oehl, F., V. M. Santos y J. Palenzuela. 2016. Paraglomus turpe, a new arbuscular mycorrhizal fungal species from Central European agricultural soils. Nova Hedwigia 103(3): 491-499. DOI: https://doi.org/10.1127/nova hedwigia/2016/0367

Oehl, F., J. Palenzuela, I. Sánchez-Castro, P. Kuss, E. Sieverding y G. Alves da Silva. 2012. Acaulospora nivalis, a new fungus in the Glomeromycetes, characteristic for high alpine and nival altitudes of the Swiss Alps. Nova Hedwigia 95(1): 105-122. DOI: https://doi.org/10.1127/00295035/2012/0038

Oehl, F., J. Palenzuela, I. Sánchez-Castro, F. Hountondji, A. Tchabi, L. Lawouin, J. M. Barea, D. Coyne y A. G. Silva. 2011. Acaulospora minuta, a new arbuscular mycorrhizal fungal species from sub-Saharan savannas of West Africa. Journal of Applied Botany and Food Quality 84: 213-218.

Olsen S. R., C. V. Cole, F. S. Watanabe y L. A. Dean. 1954. Estimation of available phosphorus in soils by extraction with sodium bicarbonate. USDACirc 939. US Government Printing Office. Washington D.C., USA. 19 pp.

Palenzuela, J., C. Azcón-Aguilar, J. M. Barea, G. Alves da Silva y F. Oehl. 2013. Acaulospora pustulata y Acaulospora tortuosa, two new species in the Glomeromycota from Sierra Nevada National Park (southern Spain). Nova Hedwigia 97(3): 305-319. DOI: https://doi. org/10.1127/0029-5035/2013/0129

Pereira, C. M. R., B. T. Goto, D. K. Alves da Silva, A. C. Almeida de Ferreira, F. Adriano de Souza, G. Alves da Silva, L. C. Maia y F. Oehl. 2015. Acaulospora reducta sp. nov. and A. excavata-two glomeromycotan fungi with pitted spores from Brazil. Mycotaxon 130(4): 983-995. DOI: https://dx.doi.org/10.5248/130.983 
Pereira, C. M. R., L. C. Maia, I. Sánchez-Castro, J. Palenzuela, D. K. A. Silva, R. Sudová, Z. Sýrorová, J. Rydlová, M. Čtvrtliková, B. T. Goto, G. Alves da Silva y F. Oehl. 2016. Acaulospora papillosa, a new mycorrhizal fungus from NE Brazil, and Acaulospora rugosa from Norway. Phytotaxa 260(1): 014-024. DOI: https://dx.doi. org/10.11646/phytotaxa.260.1.2

Posada, R. H., M. Sánchez de Prager, G. Heredia-Abarca y E. Sieverding. 2016. Effects of soil physical and chemical parameters, and farm management practices on arbuscular mycorrhizal fungi communities and diversities in coffee plantations in Colombia and Mexico. Agroforestry Systems 92(2): 555-574. DOI: https://doi.org/10.1007/ s10457-016-0030-0

Robles-Martínez, M. L., C. Robles, F. Rivera-Becerril, P. Ortega-Larrocea y L. Pliego-Marín. 2013. Inoculación con consorcios nativos de hongos de Micorriza arbuscular en Agave angustifolia Haw. Revista Mexicana de Ciencias Agrícolas 6: 1231-1240.

Schüßler, A. 2017. Glomeromycota: species list. http://www. amf-phylogeny.com/ (consultado diciembre de 2017).

Stutz, J. C. y J. B. Morton. 1996. Successive pot cultures reveal high species richness of arbuscular endomycorrhizal fungi in arid ecosystems. Canadian Journal of Botany 74(12): 1883-1889. DOI: https://doi.org/10.1139/b96-225 Trinidad-Cruz, J. R., E. E. Quiñones-Aguilar, L. V. HernándezCuevas, L. López-Pérez y G. Rincón-Enríquez. 2017. Hongos micorrízicos arbusculares asociados a la rizosfera de Agave cupreata en regiones mezcaleras del estado de Michoacán, México. Scientia Fungorum 45: 13-25.

Van Reeuwijk, L. P. 1999. Procedimientos para análisis de suelos (clasificación y correlación). In: GutiérrezCastorena, M. C., C. A. Taveres-Espinoza y C. A. OrtizSolorio (traductores). Traducción al español. Especialidad de Edafología, Colegio de Postgraduados. Montecillo, México. 145 pp.

Varela-Fregoso, L., A. Mora-Velázquez, C. G. ChávezHernández, A. Martínez-Bernal, R. García-Sánchez, E. Chimal-Sánchez y N. M. Montaño. 2017. Acaulospora alpina y Ambispora fennica, dos registros nuevos de hongos micorrizógenos arbusculares para México. Revista Mexicana de Biodiversidad 88(3): 496-501. DOI: https://dx.doi.org/10.1016/j.rmb.2017.06.005

Vázquez-Pérez, N. 2015. Variación morfológica y genética de Agave karwinskii (Agavaceae) en los estados de Oaxaca y Puebla. Tesis de maestría. Instituto de Biología, Universidad Nacional Autónoma de México. Cd. Mx., México. 81 pp.

Violi, A. H., A. F. Barrientos-Priego, S. F. Wright, E. EscamillaPrado, J. B. Morton, J. A. Menge y C. J. Lovatt. 2008. Disturbance changes arbuscular mycorrhizal fungal phenology and soil glomalin concentrations but not fungal spore composition in montane rainforests in Veracruz and Chiapas, Mexico. Forest Ecology and Management 254(2): 276-290. DOI: https://dx.doi.org/10.1016/j. foreco.2007.08.016 